\title{
Aerodynamic Effects of Uniform Blowing and Suction on a NACA4412 Airfoil
}

\author{
Marco Atzori ${ }^{1,2} \cdot$ Ricardo Vinuesa $^{1,2} \cdot$ Georg Fahland $^{3} \cdot$ Alexander Stroh $^{3}$. \\ Davide Gatti $^{3}$ - Bettina Frohnapfel ${ }^{3}$. Philipp Schlatter ${ }^{1,2}$
}

Received: 7 November 2019 / Accepted: 2 April 2020 / Published online: 23 April 2020

(c) The Author(s) 2020

\begin{abstract}
We carried out high-fidelity large-eddy simulations to investigate the effects of uniform blowing and uniform suction on the aerodynamic efficiency of a NACA4412 airfoil at the moderate Reynolds number based on chord length and incoming velocity of $R e_{c}=200,000$. We found that uniform blowing applied at the suction side reduces the aerodynamics efficiency, while uniform suction increases it. This result is due to the combined impact of blowing and suction on skin friction, pressure drag and lift. When applied to the pressure side, uniform blowing improves aerodynamic efficiency. The Reynoldsnumber dependence of the relative contributions of pressure and friction to the total drag for the reference case is analysed via Reynolds-averaged Navier-Stokes simulations up to $R e_{c}=10,000,000$. The results suggest that our conclusions on the control effect can tentatively be extended to a broader range of Reynolds numbers.
\end{abstract}

Keywords Active flow control · Turbulence simulation · Wing section · Aerodynamics

\section{Introduction}

The scientific community is devoting a significant effort to develop drag-reduction techniques, which would potentially be highly beneficial to e.g. reduce the consumption of fossil fuels. In the case of airplanes, which constitute a relevant portion of the greenhousegases emission from the transportation sector (Banister et al. 2011), the drag consists of mainly two different contributions. They are the lift-induced $(\simeq 35 \%)$ and viscous drag ( $\simeq 55 \%$ ), which together amounts to roughly $90 \%$ of the total drag, according to industrial estimates (Wood 2004; Schrauf 2005). The continuous design optimisation carried out over the past decades led to sizeable reductions of lift-induced drag. Thus, further reductions of this contribution to the total drag probably require the introduction of new innovative

Marco Atzori

atzori@mech.kth.se

1 Linné FLOW Centre, KTH Mechanics, 10044 Stockholm, Sweden

2 Swedish e-Science Research Centre (SeRC), Stockholm, Sweden

3 Institute of Fluid Mechanics, Karlsruhe Institute of Technology (KIT), Karlsruhe, Germany 
designs (Abbas et al. 2013). In comparison, less success has been achieved in reduction of viscous drag resulting from the shear stress of the fluid which yields both friction drag, i.e. the friction force on the body surface, and to pressure drag from the developing boundary layer. The occurrence of flow separation, in which the streamlines are deflected away from the aerodynamic body, is also a factor that can significantly contribute to increasing pressure drag.

Many studies reported that it is theoretically possible to control the flow in order to effectively diminish the skin friction while gaining a net-energy saving, proposing a variety of different strategies. These include passive methods, such as riblets (Bechert and Bartenwerfer 1989; Choi et al. 1993), a drag-reducing surface structuring proven successful on passenger aircrafts (Viswanath 2002), as well as active ones, in which the drag reduction effect is achieved through an action which requires additional energy to be transferred to the flow (Gad-el Hak 2000). When the action is determined based on state of the flow, sensing is required and the control strategy is said to be reactive [see, for instance, Choi et al. (2008)], in opposition to "predetermined" strategies, for which the action is determined a priori. Predetermined strategies are particularly appealing [see, for instance, Quadrio (2011)] thanks to their comparatively low complexity and larger achievable drag reduction at the cost of sizeable power required by the control. The present work deals with the predetermined strategy for turbulent skin-friction drag reduction named "uniform blowing", which consists of the imposition of a wall-normal velocity at portions of solid boundaries.

The capability of uniform blowing to reduce skin friction has been extensively documented in both laminar and turbulent flows. Hwang (1996) carried out the first wind-tunnel experiment with the so-called micro-blowing technique (MBT). He reported that it is possible to achieve a significant drag reduction with relatively moderate blowing flow-rate if the skin friction of the perforated surface employed to perform blowing is similar enough to that of a smooth surface. He thereby conjectured that it is possible to achieve net-energy saving in real-world applications. More recent studies confirmed this possibility, investigating the effects of MBT on more complex geometries as well as on turbulent boundary layers subject to adverse-pressure gradients. For a detailed description of the development of the MBT technique, we refer to the review by Hwang (2004), whereas Kornilov (2015) discusses more recent advancements, focusing on experimental results.

High-fidelity numerical simulations could be used to better characterize the interaction between blowing and wall turbulence. However, it is still prohibitively expensive to describe the entire turbulent boundary layer together with the details of the blowing surface for reasonably high Reynolds numbers and direct numerical simulations (DNS) (Kametani and Fukagata 2011) as well as well-resolved large-eddy simulation (LES) (Kametani et al. 2015) are often employed with the aid of a simplification, namely that appropriate Dirichlet boundary conditions can model the effect of MBT. It is important to note that such a simplification artificially reduces the parameter space for uniform blowing and suction to control location and intensity. In a real-case scenario, other aspects such as the orifice geometry, arrangement, and orientation will play a role, as well as the dynamic interactions between the jets and the external flow. Nevertheless, in the present study aimed at fundamental considerations, the representation of blowing and suction via a Dirichlet boundary condition is maintained.

To the authors' knowledge, the first numerical study investigating a turbulent boundarylayer flow subjected to blowing is that of Park and Choi (1999), who employed DNS and considered turbulent boundary layers (TBL) at the low Reynolds number based on the displacement thickness and free-stream velocity of 500. They applied blowing (and suction) 
from a spanwise "slot" with a streamwise width of approximately 125 viscous wall units with relatively intense blowing, i.e. from $\simeq 2$ to $\simeq 9 \%$ of the freestream velocity. However, these authors did not report in detail the skin-friction reduction at the control location since their main focus was to study the downstream effects of blowing and suction. Moreover, their conclusion that blowing increases the skin friction downstream of the inject location does not hold for higher Reynolds numbers (Stroh et al. 2016) and it is probably due to low-Reynolds-number effects.

In 2011, Kametani and Fukagata (2011) performed DNS of a TBL with blowing and suction at Reynolds numbers based on the momentum thickness between $\operatorname{Re}_{\theta}=300$ and $R e_{\theta}=700$, with intensities up to $1 \%$ of the free-stream velocity $U_{\infty}$. They also consider the energy input associated with uniform blowing to estimate the upper bound of control efficiency and confirmed that it is theoretically possible to achieve net-energy saving. Furthermore, these authors employed the FIK identity (Fukagata and Iwamoto 2002), by means of which the skin friction is decomposed into terms related to streamwise development, mean convection, and turbulent fluctuations. They concluded that blowing results in stronger turbulence fluctuations and therefore in an increase of the corresponding contribution to skin friction. However a total skin-friction reduction is achieved because such increase is outweighed by the reduction of the contribution from mean convection normal to the wall. Uniform suction has opposite effects.

Subsequently, other numerical simulations were performed at higher Reynolds numbers, in order to assess the optimal control configuration at increasing values of $R e$, which are more relevant to the actual application of full-scale aircraft. In 2015, Kametani et al. (2015) carried out well-resolved LES of TBL at Reynolds numbers based on the free-stream velocity and the momentum thickness up to $R e_{\theta}=2500$, considering blowing and suction with an intensity of $0.1 \%$ of the free-stream velocity. In these simulations, the boundary layer was initialised with a Blasius profile at the inlet of the computational domain, and transition to turbulence is induced with tripping, leading to fully-developed turbulence for $R e_{\theta}>500$. Blowing and suction were implemented as a uniform and constant wall-normal velocity on the wall, and different control regions were considered. These authors reported achieving more than $10 \%$ drag reduction despite the relatively low blowing intensity. They also observed that the net-energy saving is larger for longer control regions, and for cases where the control is located within the earlier boundary-layer development.

Soon after, Stroh et al. (2016) studied the development of a TBL at Reynolds numbers in the range between $R e_{\theta} \simeq 500$ and 2400 downstream from the control region. They compared blowing with body force damping, the latter being a numerical control technique to model the effect of reducing near-wall fluctuations and thereby potentially affecting the skin friction. It is shown in the paper that different control techniques affect the boundarylayer development which can be described by means of a modified virtual origin. They also reported that only with uniform blowing the skin-friction reduction persists indefinitely in the region were the control is not applied, albeit it is weaker than over the control region.

Following this observation, Mahfoze et al. (2019) employed Bayesian optimization to identify the best combination of control-region length and blowing amplitude to maximize energy-saving, also including intermittent control regions. These authors also took into account the pressure measurements across the perforated plate in the experiment performed by Kornilov and Boiko (2012) to formulate a more realistic estimate of the power consumption by blowing, and they confirmed that it is possible to obtain a net-energy saving in the range of few percent.

The works mentioned so far focused on the description of the effect of blowing on developing TBLs, which remains an idealized study case. Firstly, more realistic 
scenarios exhibit more complex turbulent flows, such as TBL subjected to non-uniform pressure gradients and finite aerodynamic bodies, for which the generalization of the control techniques is not trivial. Secondly, the skin-friction reduction is beneficial in engineering applications only if it corresponds to a reduction of total drag, which also includes pressure drag, and to an improvement of the aerodynamic efficiency. For these reasons, the two following experimental works on the effects of blowing and suction on airfoils are of particular relevance. On the one hand, Eto et al. (2019) considered blowing applied over the suction side of a Clark-Y airfoil at a Reynolds number based on the chord length of $R e_{c}=1,600,000\left(R e_{c}=U_{\infty} c / v\right.$, where $c$ is the chord length and $v$ the kinematic viscosity) and a blowing intensity of $0.14 \% U_{\infty}$, and they observed a local reduction of the skin friction between $\simeq 20$ and $\simeq 40 \%$. However, they also reported that the total drag, estimated from the pressure measurements in the wake of the airfoil, increased. On the other hand, Kornilov et al. (2019) carried out experiments on a NACA 0012 airfoil at $R e_{c}=700,000$, applying blowing and suction over both sides of the airfoil between $0.623 c$ and $0.775 c$ from the leading edge. They confirmed that blowing over the suction side does not reduce the total drag, but they also observed that blowing over the pressure side and suction over suction side have a beneficial effect, achieving a reduction of total drag of the order of $10 \%$.

The experimental results discussed above highlight the need of adopting more realistic benchmarks to assess the potential of improving the aerodynamic efficiency by employing uniform blowing and suction. This necessity is the motivation of the present study, which extends preliminary work using LES to investigate blowing over the suction side of a NACA4412 at a Reynolds number of $R e_{c}=100,000$ (Vinuesa and Schlatter 2017). We performed well-resolved LES of the incompressible flow around a NACA4412 airfoil at a Reynolds number of $R e_{c}=200,000$ and angle of attack $(A o A)$ of 5 degrees, considering several control configurations, including both uniform blowing and uniform suction. Furthermore, since blowing and suction affect in opposite ways skin-friction and pressure drag, we performed a series of Reynolds-averaged Navier-Stokes (RANS) simulations for the airfoil without control, in order to describe how the relative contributions to the total drag vary with $R e$. Note that this is the first time that control using blowing and suction is investigated over a wing section using high-fidelity numerical simulations. The only previous study pertaining control effects on a similar geometry is that of Albers et al. (2019), who performed well-resolved LES of a DRA2303 wing section at $R e_{c}=400,000$ with spanwise travelling waves which is conceptually different control mechanism than uniform blowing or suction. An important point that we do not discuss is the possible net-energy saving. This is due to the fact that in the case of uniform blowing and suction the input power consumption strongly depends on the actual (engineering) way in which the control is implemented and therefore requires certain assumptions. Most idealisations, such as considering an arbitrary pressure difference, may deliver unrealistic results which strongly overestimate the energy saving. A detailed investigation of the energy losses in a realistic implementation deserves a comprehensive description, which is out of the scope of the present study.

The paper is organized as follows: in Sect. 2, we describe the cases considered in this study and the numerical setup; in Sect. 3, we present the results regarding the control effects on skin-friction, total drag, aerodynamic efficiency, and inner-scaled velocity profiles and the evolution of skin-friction and pressure drag for increasing Reynolds numbers; while Sect. 4 contains the concluding discussion. In the "Appendix", we discuss the statistical convergence of the results and we compare the momentum balance within a control volume with the total forces integrated over the airfoil surface. 


\section{Methodology}

\subsection{Considered Cases}

We present a relatively large set of simulations, which are listed in Table 1 . Due to the exploratory nature of the project, we selected the considered cases in a step-by-step process, following the most promising path for our goals.

The reference case (Case A), is a NACA4412 airfoil at a chord Reynolds number of 200, 000 and angle of attack of 5 degrees. The airfoil and angle of attack are the same as in the study by Vinuesa et al. (2018), who characterized the TBL over a NACA4412 airfoil at Reynolds numbers between $R e_{c}=100,000$ and $R e_{c}=1,000,000$. The NACA4412 airfoil is considered because the pressure distribution over the suction side is weakly dependent on the Reynolds number (Pinkerton 1938). The choice of AoA serves the purpose of giving a relatively high adverse pressure gradient on the suction side, without leading to mean separation. The choice of the Reynolds number is the results of a compromise between the need of studying a well-developed TBL and the computational cost.

In Cases $\mathrm{B}$ and $\mathrm{C}$, uniform blowing has been applied over the suction side of the airfoil from $x / c=0.25$ to $x / c=0.86$, with intensities $0.1 \%$ and $0.2 \%$ of the incoming flow, respectively. The region where the control is applied is the same as in a preliminary study (Vinuesa and Schlatter 2017), and it is limited in the streamwise direction to reduce the risk of separation in the region of strong adverse pressure gradient (APG). Due to the observation that uniform blowing over the suction side decreases the aerodynamics efficiency, in Cases D and E we applied uniform suction over the same region and with the same intensities as those in cases B and C, respectively. In Case F, we applied both uniform suction over the suction side and uniform blowing over the pressure side with intensity $0.1 \% U_{\infty}$, again between $x / c=0.25$ and $x / c=0.86$. We chose this configuration because it yields zero mass flux over the airfoil surface, which may be a beneficial condition in realistic application scenarios.

Case F leads to the observation that blowing has a beneficial effect if applied over the pressure side. Therefore, in Cases $\mathrm{G}$ and $\mathrm{H}$, we applied blowing over the pressure side with an intensities of $0.1 \% U_{\infty}$ and $0.2 \% U_{\infty}$, respectively. Since for these blowing intensities there is no risk of inducing separation over the pressure side, we considered the extended control region from $x / c=0.2$ to $x / c=1.0$ for these cases.

Table 1 Control configurations described in the present paper

\begin{tabular}{|c|c|c|c|}
\hline Case & Control over the suction side & Control over the pressure side & Color \\
\hline $\mathrm{A}$ & - & - & \\
\hline $\mathrm{B}$ & U.B., $0.1 \% U_{\infty}(0.25<x / c<0.86)$ & - & \\
\hline $\mathrm{C}$ & U.B., $0.2 \% U_{\infty}(0.25<x / c<0.86)$ & - & \\
\hline $\mathrm{D}$ & U.S., $0.1 \% U_{\infty}(0.25<x / c<0.86)$ & - & \\
\hline $\mathrm{E}$ & U.S., $0.2 \% U_{\infty}(0.25<x / c<0.86)$ & - & \\
\hline $\mathrm{F}$ & U.S., $0.1 \% U_{\infty}(0.25<x / c<0.86)$ & U.B., $0.1 \% U_{\infty}(0.25<x / c<0.86)$ & \\
\hline $\mathrm{G}$ & - & U.B., $0.1 \% U_{\infty}(0.20<x / c<1.00)$ & \\
\hline $\mathrm{H}$ & - & U.B., $0.2 \% U_{\infty}(0.20<x / c<1.00)$ & \\
\hline
\end{tabular}

"U.B." and "U.S." are uniform blowing and uniform suction, respectively. For all the cases, $R e_{c}=200,000$ and $A o A=5$ degrees 
Figure 1 illustrates the effects of uniform blowing and uniform suction over the instantaneous streamwise component of the velocity in Cases $\mathrm{C}$ and $\mathrm{E}$. It is visually apparent that blowing and suction affects the boundary-layer thickness in addition to the skin friction.

\subsection{Large-Eddy Simulations (LES)}

We performed LES using the spectral-element code Nek5000 (Fischer et al. 2008). The numerical setup is similar to that already described in Ref. Vinuesa et al. (2018). In these simulations, the domain is divided into elements, and velocity and pressure are represented inside each element by Lagrange interpolants. Following the $P_{N} P_{N-2}$ formulation (Maday and Patera 1984), if the polynomial order is $P=N-1$, the velocity is defined on $N^{3}$ points per element distributed according to the Gauss-Lobatto-Legendre (GLL) quadrature rule, and the pressure is defined on a staggered grid of $(N-2)^{3}$ points per element with the Gauss-Legendre distribution (GL). The incompressible Navier-Stokes equations are advancing in time, with the non-linear term being treated by an explicit third-order
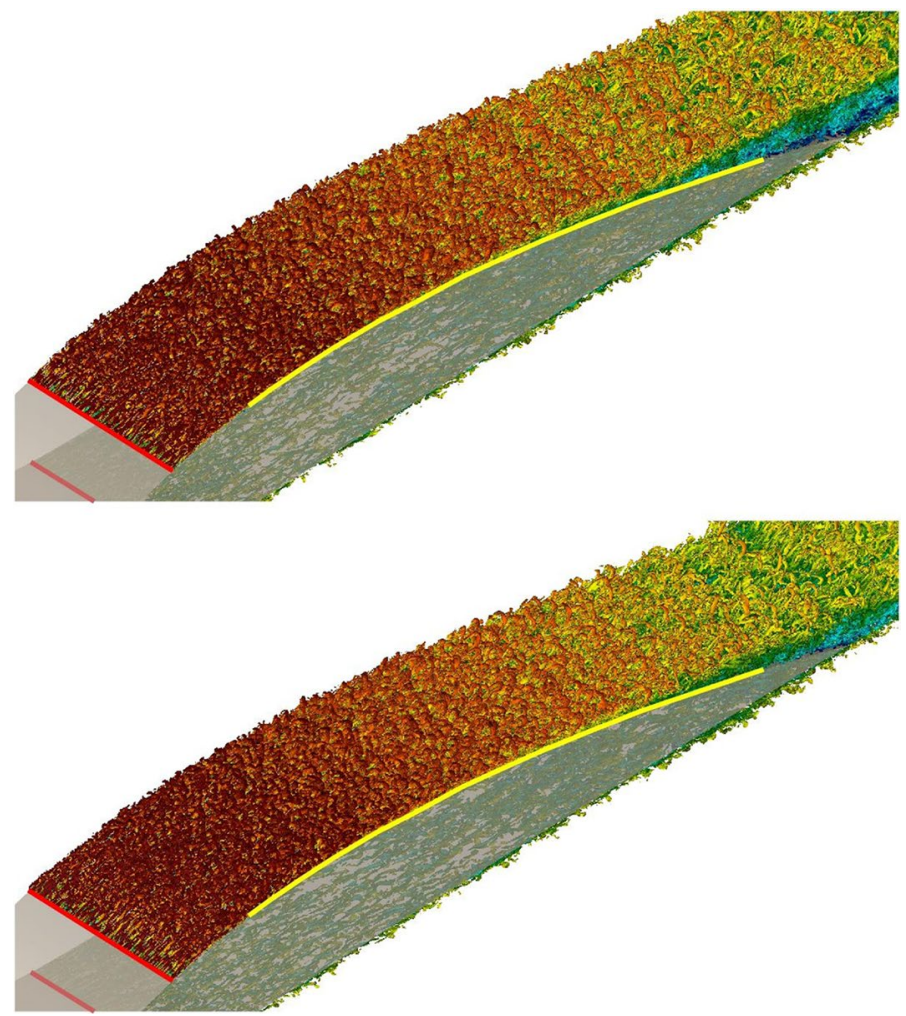

Fig. 1 Vortex clusters identified with the $\lambda_{2}$ criterion (Jeong and Hussain 1995) in Cases (top) C and (bottom) E, with blowing and suction applied over the suction side, respectively. The differences between the two cases are visually apparent only in the vicinity of the trailing edge. Vortex clustered coloured with the instantaneous velocity component, from (red) $u \approx 1.7$ to (blue) $u \approx-0.2$. The yellow and red lines indicate the spanwise controlled region and the tripping location, respectively. Note that the tripping is applied over both sides of the airfoil 
extrapolation, and the viscous term with a third-order implicit backward differencing formula (BDF). Pressure and velocity are decoupled using a time-splitting operation (Fischer 1997).

The domain size is $6 c \times 4 c \times 0.2 c$ in the horizontal, vertical and spanwise directions, respectively, with periodic boundary conditions in the third direction. Note that the leading and trailing edge of the airfoil are located $2 c$ and $3 c$ from the front and rear boundaries of the domain, respectively. The boundary conditions for the front, upper and lower limits of the domain are of Dirichlet type, prescribing a far-field velocity distribution estimated with an auxiliary RANS simulation. The RANS simulation is performed employing the $k-\omega$ SST model (Bottaro et al. 1994) in a computational domain that extends up to 200c far from the airfoil in each direction. The boundary condition for the outlet is the one proposed by Dong et al. (2014). Blowing and suction are implemented as boundary conditions on the airfoil surface. Exploiting the local unity vector normal to the surface and pointing in the flow domain, the wall-normal velocity to be imposed is decomposed into its Cartesian components, which are imposed as Dirichlet boundary condition. The RANS boundary conditions are the same for all the cases in the present study. We considered employing RANS with control for the control cases, but we found that the control effects, at the location of the boundaries, are small and comparable with the differences between RANS and LES in the reference case. Note that the discrepancy between the RANS and LES in the mean velocity at the far-field is below $1 \%$. Therefore, we avoid including blowing/suction in the auxiliary RANS simulation to avoid introducing additional uncertainty.

To reduce the computational cost of the study, we performed well-resolved LES based on a relaxation-term filter (Schlatter et al. 2004). This formulation is in principle equivalent to explicitly filtering the highest spectral modes but, since the filtering operation is performed implicitly through a volume force, it has the advantages of preserving continuity and being independent on the time step. Therefore, the set of equations that are solved are:

$$
\begin{aligned}
& \frac{\partial u_{i}}{\partial t}+u_{j} \frac{\partial u_{i}}{\partial x_{j}}=-\frac{1}{\rho} \frac{\partial p}{\partial x_{i}}+v \frac{\partial^{2} u_{i}}{\partial x_{j} \partial x_{j}}-\mathcal{H}\left(u_{i}\right) \\
& \frac{\partial u_{i}}{\partial x_{i}}=0
\end{aligned}
$$

where $\mathcal{H}\left(u_{i}\right)$ is a high-pass filter that acts on a certain number of modes and with a certain strength, and both parameters require calibration. Note that repeated indexes imply summation.

We designed the mesh considering the results of the validation of the SGS model performed by Negi et al. (2018), who compared DNS and LES results from a simulation of a NACA4412 airfoil at $R e_{c}=400,000$, showing very good agreement between both. Following the same guideline, the grid spacing in the turbulent-boundary-layer region in the proximity of the airfoil surface is $\Delta x_{t}^{+}=18, \Delta y_{n}^{+}=(0.64,11)$ and $\Delta z^{+}=9$ respectively in the wall-normal, wall-tangential and spanwise directions. Note that the viscous length is $l^{*}=v / u_{\tau}$, where the friction velocity is $u_{\tau}=\sqrt{\tau_{w} / \rho}$, the wall-shear stress is $\tau_{w}=\rho v\left(\mathrm{~d} U_{t} / \mathrm{d} y_{n}\right)_{y_{n}=0}$ and $\rho$ is the fluid density. In this study, we employed approximately 127,000 spectral elements and the 11 th polynomial order, leading to a number of grid points of approximately 220 millions.

We applied tripping of the boundary layer via a localized body force over both the suction and the pressure sides of the airfoil (the upper and lower sides, respectively) at a distance from the leading edge of $x / c=0.1$. The body force is designed to emulate the effect of the devices usually employed in experimental facilities, as described by Schlatter and 
Örlü (2012). To minimize transient times, we adopt the following procedure: (1) the initial conditions are the velocity and pressure fields obtained from the same RANS solution employed for the boundary condition; (2) we start the simulation at polynomial order $P=5$ and we progressively increase it, up to $P=11$, running 4 and 2 flow-over times at $P=5$ and $P=7$, respectively; (3) once $P=11$ is reached, we start sampling statistics after 2 additional flow-over times. Note that 1 flow-over time is the time needed for a fluid particle moving with the incoming velocity $U_{\infty}$ to travel a distance of $c$. For the cases with control, we start the simulation from a fully-developed turbulent field of the reference case, and we monitor the time evolution of lift and drag to identify when the statistically stationary state is reached.

Adopting the aforementioned procedure, each case requires between $\simeq 10$ and $\simeq 15$ flow-over times to obtain converged statistics, including the components of the Reynolds stress and the terms relevant for the turbulent kinetic energy budget (not shown here). However, we used a shorter average time for few cases, since it is sufficient to obtain an estimate of the aerodynamic efficiency. A more detailed assessment of the convergence is reported in the "Appendix". The approximate computational cost to simulate 10 flow-over times is 1 million CPU hours on a Cray-XC 40 system.

\subsection{Reynolds-Average Navier-Stokes (RANS) Simulations}

Two-dimensional RANS simulations were carried out to assess the $R e$-dependence of pressure and friction contributions to total drag over the Reynolds-number range from 200, 000 to $10,000,000$, which would be otherwise computationally unaffordable with present wallresolved LES. The results are leveraged to extrapolate the effect of the control observed at lower values of $R e$ via LES to higher Re flows. We employ the $k-\omega$ SST turbulence model (Menter et al. 2015) and the steady state solver simpleFoam from the OpenFOAM CFD-Toolkit (Foundation 2019) with a specific adjustment to enable imposing the same transition location as in LES. Hereby the turbulent kinetic energy $k$ is kept at zero until $x=0.09 c$, followed by the tripping location at $x_{t r}=0.1 c$. There, a small source term for the turbulent kinetic energy $k$ is applied at a distance from the wall equal to the local displacement thickness $\delta^{*}$. This is necessary as the standard production term for $k$ would not be sufficient in order to get an immediate transition, in particular at such small $R e_{c}$. This setup provides similar results to the use of the $\gamma-R e_{\theta}$ transitional turbulence model (Menter et al. 2006), except for the transition prediction. The additional source term is adjusted to fit the typical friction-coefficient distribution at transition location observed in the ERCOFTAC T3 test cases (Savill 1992). Even though these cases are related to bypass transition and not trip-induced transition, the resulting shear-stress curves are adequate for the present purpose. A two-step solution approach was taken in order to reduce calculation effort: first, an initial solution per mesh was created with residuals to reach levels below $10^{-5}$. The final solution for a specific parameter set included the condition of residuals to drop below $10^{-6}$. The computational grid is a $2 \mathrm{D}$ structured $\mathrm{C}-\mathrm{Mesh}$ with a radius of $r=50 c$ and an outlet distance of $d_{O}=75 c$. The location of the first grid point in the wallnormal direction satisfies $y^{+}<1$, and we consider a wall-normal expansion rate of 1.1. This is ensured by calculating approximate boundary-layer properties for the specific airfoil and the expected operating point ( $R e, \alpha$, tripping position) using the boundary element solver XFOIL (Drela 1989) before mesh generation. For the present calculations this means that there is one mesh per $R e$. The wall-parallel refinement depends on the wall curvature but is never greater than $\Delta x=5 \cdot 10^{-3} c$ at the wall. The inflow boundary condition for the 
velocity is a fixed value corresponding with a steady incoming flow. At the outflow, homogeneous Neumann boundary conditions are applied to all flow quantities. The boundary conditions of the pressure field are set similar to the velocity. A freestreamPressure-condition is applied to inlet and outlet. This results in a fixed-value Dirichlet condition where the flow vector points into the domain and a vanishing gradient otherwise. On the airfoil surface, the vanishing gradient is also applied. On the the surface of the airfoil usual wall boundary conditions are applied with $k$ forced towards zero to assure numerical stability. For $\omega$ the method proposed by Menter (1994) is used defining a wall function which sets the value for $\omega$ in the first cell above the wall depending on its wall-normal distance. This is the numerical equivalent to imposing $\omega \rightarrow \infty$ for vanishing wall-normal distance.

\section{Results}

In this section, we first describe the control effects on the local skin friction and its relative reduction/increase. Then, we consider the "side" effects of uniform blowing and suction on pressure drag and thus their impact on total drag. Additionally, a detailed description of the modification of the viscous-scaled mean velocity profiles due to the control is provided. Lastly, we study relative share of pressure and skin friction to total drag as a function of $R e$, comparing well-resolved LES and RANS simulations.

\subsection{Local Skin Friction}

Skin-friction reduction is usually the main goal of applying uniform blowing. The skinfriction coefficient, defined as $c_{f}=\tau_{w} /\left(\frac{1}{2} \rho U_{\infty}^{2}\right)$, is shown for the different cases in Fig. 2, for the suction (left) and the pressure sides (right). Note that the local $c_{f}$ is usually defined in terms of the local velocity at the boundary-layer edge if the main focus is to study the
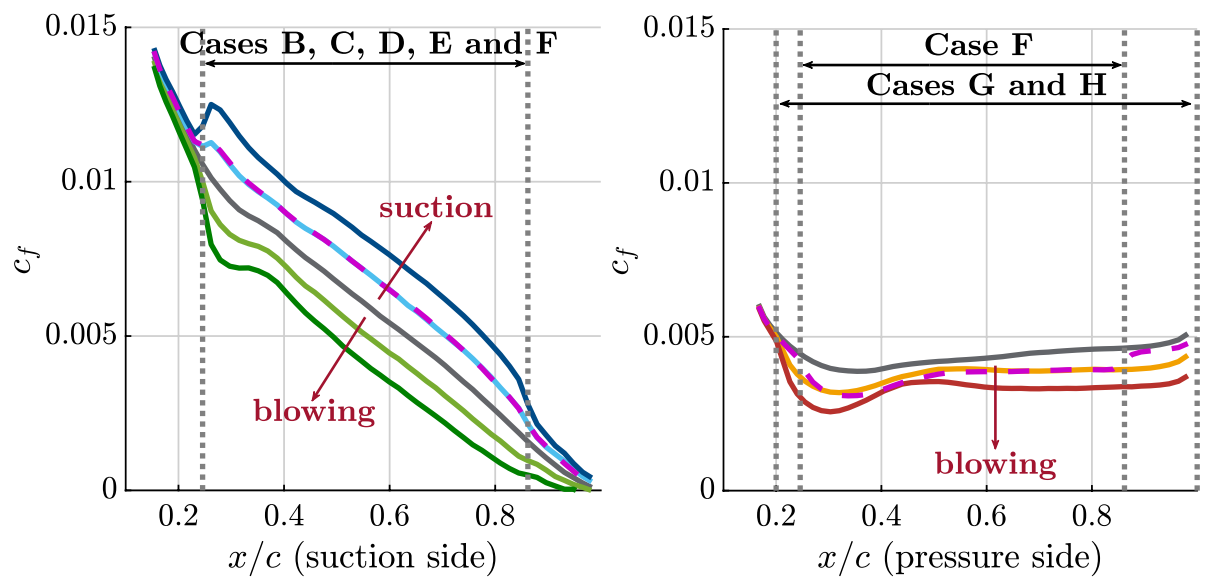

Fig. 2 Local skin-friction coefficient for (left) suction side for Cases A, B, C, D, E, and F and (right) pressure side for Cases A, F, G and H. Color code as in Table 1. Note that the magenta and cyan lines on the left (Cases D and E, respectively) and the magenta and orange lines on the right (Cases D and F, respectively) are overlapping. The vertical dotted lines indicate the control region 
local properties of TBL. In this section, we employ the velocity of the incoming flow $U_{\infty}$ to properly represent the relative intensity of the skin friction with respect to the total drag.

In Case A, the streamwise development of $c_{f}$ over the suction side is a consequence of the increasingly stronger APG, which, at the present Reynolds number, leads to a state close to mean separation in the vicinity of the trailing edge. On the other hand, the moderate $c_{f}$ increase over the pressure side is due to the mild favourable pressure gradient (FPG). Over the suction side, the effects of the control are qualitatively similar to those reported when the control is applied to zero-pressure-gradient (ZPG) TBL. Both for blowing and suction, the skin-friction reduction/increase is strongest in the control region but it is also present in the downstream development of the flow. In particular, in Case $\mathrm{C}$ featuring a relatively strong blowing intensity of $0.2 \% U_{\infty}$, mean separation occurs immediately before the trailing edge, which does not occur in Case A. In all the cases, the effect of the control on $c_{f}$ is limited to the side of the airfoil where the control is applied. For instance, the $c_{f}$ curves for Cases D and F are almost identical, although in the latter blowing is applied over the pressure side. Similarly, in Cases G and H, for which the control acts only on the pressure side, $c_{f}$ over the suction side is as in the uncontrolled cases, and the same happens for Cases B, C, D and E over the pressure side (the $c_{f}$ curves which overlap with the uncontrolled case are not shown in Fig. 2). Note that the skin friction exhibits small changes upstream of the control region. This effect is caused by the introduction of a local pressure jump at the onset of the control as also observed for control onset in flat-plate turbulent boundary layers (Stroh et al. 2016). In addition, the applied control alters the (global) pressure distribution over the entire wing surface such that a slight effect of the control is present over the entire wing.

The comparison between blowing effects over suction and pressure sides unveils that the relation between those and the pressure gradient is not obvious. The blowing effects in absolute terms are stronger over the suction side than over the pressure side, which is subject to a moderate FPG. However, the reduction (increase) due to blowing (suction) is almost constant over the control region, despite the increasing APG. Furthermore, in Case F, contrary to what happens for ZPG and APG, the still moderate FPG prevents the effects of blowing from extending to the region downstream the control.

To better characterize the alteration of skin friction, we also consider the relative reduction of the wall-shear stress with respect to the reference case, defined as $R=1-\tau_{w} / \tau_{w, 0}$ (see Fig. 3). Over the suction side, the fact that the absolute reduction of the skin friction is almost constant, while the skin friction itself reduces, determines that $R$ increases in this region. In particular, despite the fact that the differences between controlled and reference values are smaller downstream of the control region, $R$ eventually becomes higher than in the control region close to the trailing edge. In the case of blowing, $R=+1$ corresponds to the location of mean separation, which occurs approximately at the trailing edge for Case $\mathrm{B}$ and at $x / c=0.94$ for Case $\mathrm{C}$. In the control region, $R$ varies between +10 and $+42 \%$ for Case B and between +20 and $+70 \%$ for Case C. For Cases D and E, the skin friction increases and $R$ ranges between -11 and $-42 \%$, and between -24 and $-90 \%$, respectively. For both blowing and suction, $R$ also shows small variations in the region before the position of the maximum camber $(x / c=0.4)$, while in the streamwise region $0.4<x / c<0.86$, i.e. the portion of the control area subjected to an increasing adverse pressure gradient, $|R|$ increases. Farther downstream, its behaviour is more complex, a fact which is due to the very low value of $C_{f}$ in the reference case. The behavior of $R$ for the control region over the pressure side is different than over the suction side, due to the different behavior of $c_{f}$ and the effects of the FPG. For $x / c<0.4$, a region where $c_{f}$ itself is lower than for the suction side, $R$ is higher, reaching $+20 \%$ and $+34 \%$ for Cases $\mathrm{G}$ and $\mathrm{H}$, respectively. However, 

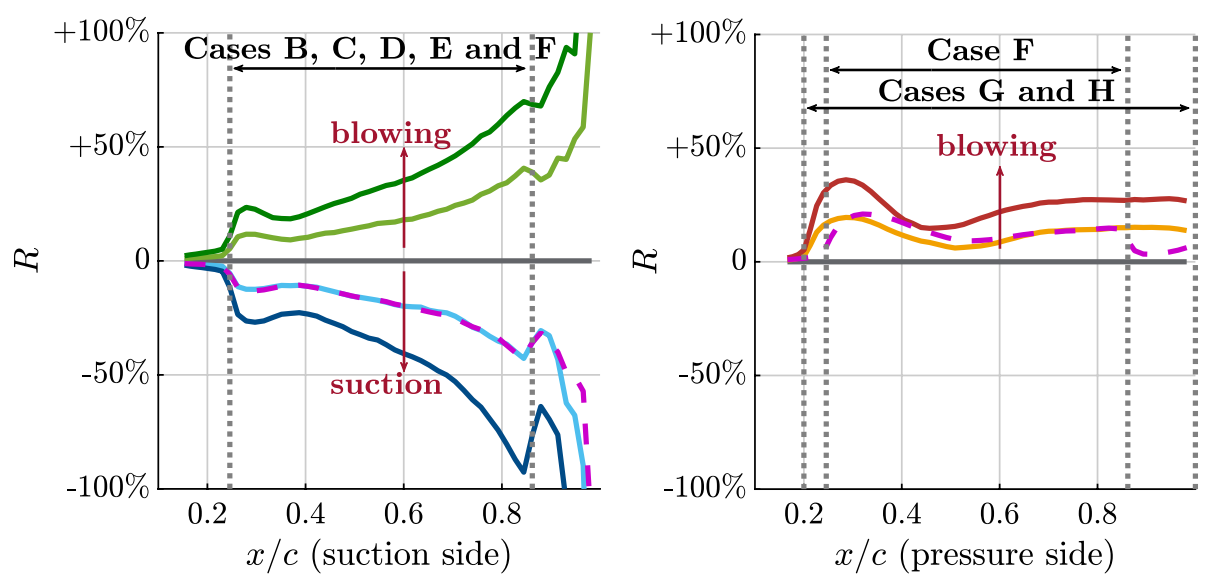

Fig. 3 Relative change of wall-shear stress over (left) the suction side in Cases A, B, C, D, E, and F, and (right) the pressure side in Cases A, F, G and H. Color code as in Table 1

downstream that region the blowing effects are smaller than over the suction side due to the FPG and so is $R$. For instance, at $x / c=0.8$, in Case $\mathrm{G}$ we observe $R=14 \%$ over the pressure side, to be compared with $R=33 \%$ over the suction side in Case B; note that for Cases $\mathrm{G}$ and $\mathrm{B}$ the blowing intensity is $0.1 \% U_{\infty}$. The comparison between Cases $\mathrm{F}$ and $\mathrm{G}$, which have the same blowing intensity over the suction side but different control regions, is also interesting. In Case F, for which the control region begins further downstream than in Case $\mathrm{G}$, the highest value of $R$ also moves downstream, which follows the fact that the history of the flow influences its local properties (Bobke et al. 2017). However, further downstream, between approximately $x / c=0.6$ and $x / c=0.8, R$ (and $c_{f}$ ) for Cases $\mathrm{F}$ and $\mathrm{G}$ are not distinguishable.

To summarize, we observed that uniform blowing and uniform suction, respectively, decreases and increases the skin friction, in agreement with the numerous studies on the topic. Moreover, the effect of blowing and suction depends on the pressure gradient acting on the TBL. It is stronger for the APG TBL developing over the suction side and progressively intensifies as the APG becomes more intense. The next step is to discuss the impact of the control on the total drag and the overall aerodynamic efficiency.

\subsection{Total Drag and Aerodynamic Efficiency}

The aerodynamic efficiency for an airfoil is defined as the ratio between the lift force, $f_{l}$, and the drag force, $f_{d}$, per unit length, which are usually expressed in terms of lift and drag coefficients, $C_{l}=f_{l} /(q c)$ and $C_{d}=f_{d} /(q c)$, respectively, where $q=\frac{1}{2} \rho U_{\infty}^{2}$ is the reference dynamic pressure. The lift and drag forces are the two components of the total force integrated over the perimeter of the airfoil and projected along the directions perpendicular and parallel to the incoming flow. The total force is then the sum of two contributions, namely the viscous force and the pressure force. From the average fields obtained through a numerical simulation, we can compute $f_{l}$ and $f_{d}$ directly as follows: 

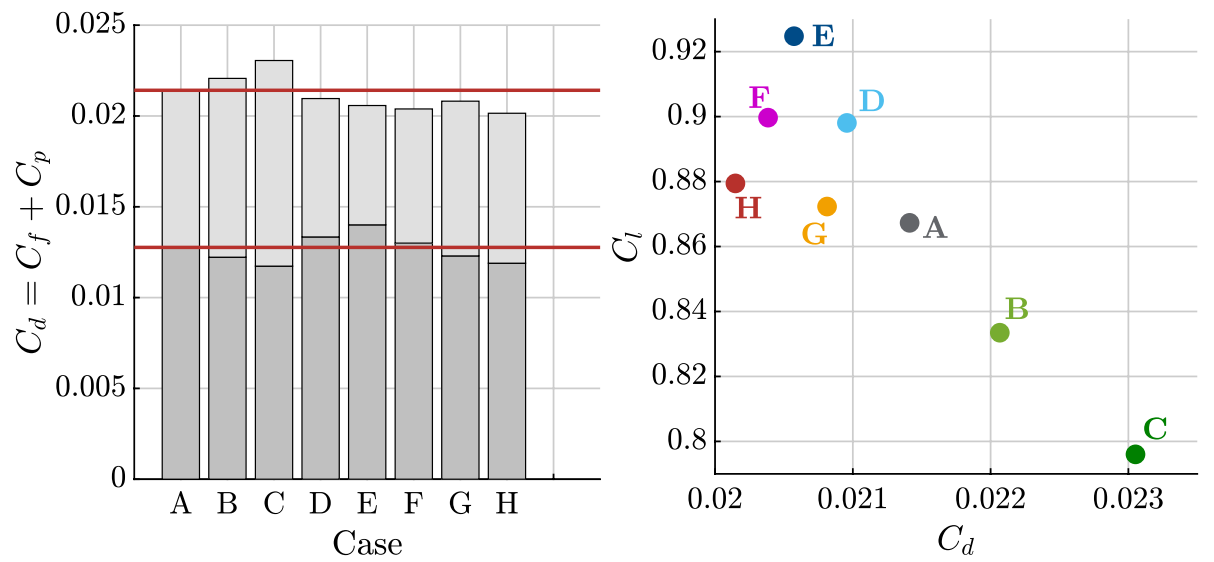

Fig. 4 (Left) Total drag for all the considered cases, where dark and light grey represent the skin-friction and the pressure contributions to the drag, respectively. The horizontal red lines mark the values of the skin friction and the total drag in the uncontrolled case. (Right) Relation between the lift and drag for all the cases. Color code as in Table 1

Table 2 Control effects on the integrated lift $\left(C_{l}\right)$, skin-friction $\left(C_{d, f}\right)$, pressure $\left(C_{d, p}\right)$, total drag $\left(C_{d}\right)$, as well as aerodynamic efficiency $(L / D)$. The values in parenthesis are the relative change with respect to Case A

\begin{tabular}{lccccc}
\hline Case & $C_{l}$ & $C_{d, f}$ & $C_{d, p}$ & $C_{d}=C_{d, f}+C_{d, p}$ & $L / D$ \\
\hline A & 0.867 & 0.0128 & 0.0087 & 0.0215 & 41 \\
B & $0.833(-4 \%)$ & $0.0122(-4 \%)$ & $0.0099(+14 \%)$ & $0.0221(+3 \%)$ & $38(-7 \%)$ \\
C & $0.796(-8 \%)$ & $0.0117(-8 \%)$ & $0.0113(+31 \%)$ & $0.0231(+8 \%)$ & $34(-15 \%)$ \\
D & $0.898(+4 \%)$ & $0.0133(+4 \%)$ & $0.0076(-12 \%)$ & $0.0210(-2 \%)$ & $43(+6 \%)$ \\
E & $0.925(+7 \%)$ & $0.0140(+10 \%)$ & $0.0066(-24 \%)$ & $0.0206(-4 \%)$ & $45(+11 \%)$ \\
F & $0.899(+4 \%)$ & $0.0130(+1 \%)$ & $0.0074(-14 \%)$ & $0.0204(-5 \%)$ & $44(+9 \%)$ \\
G & $0.871(+0 \%)$ & $0.0123(-4 \%)$ & $0.0084(-3 \%)$ & $0.0207(-3 \%)$ & $42(+4 \%)$ \\
H & $0.880(+1 \%)$ & $0.0119(-7 \%)$ & $0.0084(-3 \%)$ & $0.0203(-5 \%)$ & $43(+7 \%)$ \\
\hline
\end{tabular}

$$
\begin{aligned}
& f_{l}=\int_{\Xi} \tau_{w}(\hat{t} \cdot \hat{k}) \mathrm{d} \xi+\int_{\Xi}\left(p-p_{0}\right)(\hat{n} \cdot \hat{k}) \mathrm{d} \xi \\
& f_{d}=\int_{\Xi} \tau_{w}(\hat{t} \cdot \hat{i}) \mathrm{d} \xi+\int_{\Xi}\left(p-p_{0}\right)(\hat{n} \cdot \hat{i}) \mathrm{d} \xi .
\end{aligned}
$$

In these expressions, $\hat{n}, \hat{t}, \hat{i}$, and $\hat{k}$ are the unit vectors with direction normal and tangential to the airfoil surface and parallel and perpendicular to the incoming flow, respectively, $p$ is the pressure, and $p_{0}$ is the reference pressure in the freestream. The integration variable, $\xi$ is the curvilinear coordinate along the airfoil perimeter, and $\Xi$ is the perimeter length. An equivalent way to compute lift and drag is evaluating the momentum balance over a control volume surrounding the airfoil. We employed this methodology as well, finding a discrepancy of at most $0.5 \%$ for the aerodynamic efficiency $C_{l} / C_{d}$ for all the cases. A complete comparison between the results obtained with the two methods is reported in the "Appendix". The total $C_{d}$ for the different cases, decomposed into the friction contribution, $C_{d, f}$, and the pressure contribution, $C_{d, p}$, is shown in Fig. 4 (left). We also report $C_{l} / C_{d}$ as well as $C_{l}, C_{d}, C_{d, f}$ and $C_{d, p}$ in Table 2 for all the considered cases. 
It is relevant to note that, whereas the lift force is mainly due to the pressure difference between suction and pressure sides, the pressure and skin-friction contributions to the total drag have similar orders of magnitude at the present Reynolds number. For instance, for Case A, $C_{d, p}=40 \% C_{d}$ and $C_{d, f}=60 \% C_{d}$. This fact has significant consequences because uniform blowing over the suction side increases the pressure drag and this increase nullifies the skin-friction reduction and ultimately leads to higher $C_{d}$. Uniform suction has the opposite effect: the skin friction over the suction side increases, but the pressure drag sufficiently decreases to yield a lower $C_{d}$. To illustrate this phenomenon, it is worth comparing in detail the relative share of $C_{d, f}$ and $C_{d, p}$ for Cases $\mathrm{C}$ and $\mathrm{E}$ (blowing and suction over the suction side, respectively). In Case $\mathrm{C}$, both $C_{d, p}$ and $C_{d, f}$ are approximately half of the total drag $C_{d}$, which in turn is $8 \%$ higher than in Case A. On the other hand, in Case E, $C_{d, p} \approx 32 \% C_{d}, C_{d, f} \approx 68 \% C_{d}$ and the total drag is $4 \%$ lower than in the uncontrolled case. Therefore, uniform blowing leads to a higher value of the total drag and suction does the opposite as a direct consequence of the relative importance of the skin-friction and pressure contributions to drag. This fact suggests that the results may be different at higher values of the Reynolds numbers.

Remarkably, applying uniform blowing over the pressure side is not detrimental for the pressure drag for any of the configurations considered in this study, and it is always beneficial in terms of skin-friction and total drag. For instance, in Case F, the total drag is 5\% lower than in the reference case. In this case, the pressure drag is reduced by an amount very similar to that in Case $\mathrm{D}$, where only suction is applied over the suction side, but $C_{d, f}$ is lower. In Cases $\mathrm{G}$ and $\mathrm{H}$, the skin-friction reduction achieved by blowing is comparable with that of Cases B and C, but the pressure drag is slightly lower than in the reference case. Therefore, the total drag is lower in Cases $\mathrm{G}$ and $\mathrm{H}$ than in reference.

A reduction of the total drag is beneficial in terms of aerodynamic efficiency $L / D$ only if the control does not decrease lift, or if such a decrease is small enough. In Fig. 4 (right), we show $C_{l}$ as a function of $C_{d}$ for the cases considered in the present study. We observed that for all the cases where the total drag decreases, $L / D$ increases (Cases D, E, F, G and $\mathrm{H}$ ) as well, and vice-versa (Case B and C). However, for Cases $\mathrm{G}$ and $\mathrm{H}$ (uniform blowing over the pressure side), this result is due solely to the reduction of the total drag, whereas for Cases D and E (uniform suction over the suction side) it is also due to increase of lift. Such an increase in lift is also the reason why Cases $\mathrm{D}$ and $\mathrm{E}$ exhibit a higher value of $L / D$ than Cases $G$ and $H$, respectively, despite the fact that the total drag is lower in the latter.

Summarising the results, although uniform blowing over the suction side reduces the skin friction, it is detrimental for the total drag and the aerodynamic efficiency, since it increases the pressure drag and decreases lift. Uniform suction over the suction side has opposite effects, thereby increasing the aerodynamic efficiency. Uniform blowing over the pressure side reduces the skin friction, but it leaves the pressure drag and the lift almost unchanged, therefore it yields the highest aerodynamic efficiency. It is important to note that these conclusions are valid only for the range of parameters explored in the present study, since e.g. higher control intensities may qualitatively change the behaviour of the flow.

\subsection{Control Effects on Turbulence Statistics}

The effects of uniform blowing and uniform suction are well documented for canonical ZPG TBLs, but relatively little is known about their interaction with pressure gradients. In Fig. 5, we show the inner-scaled velocity profiles of the wall-tangential and 

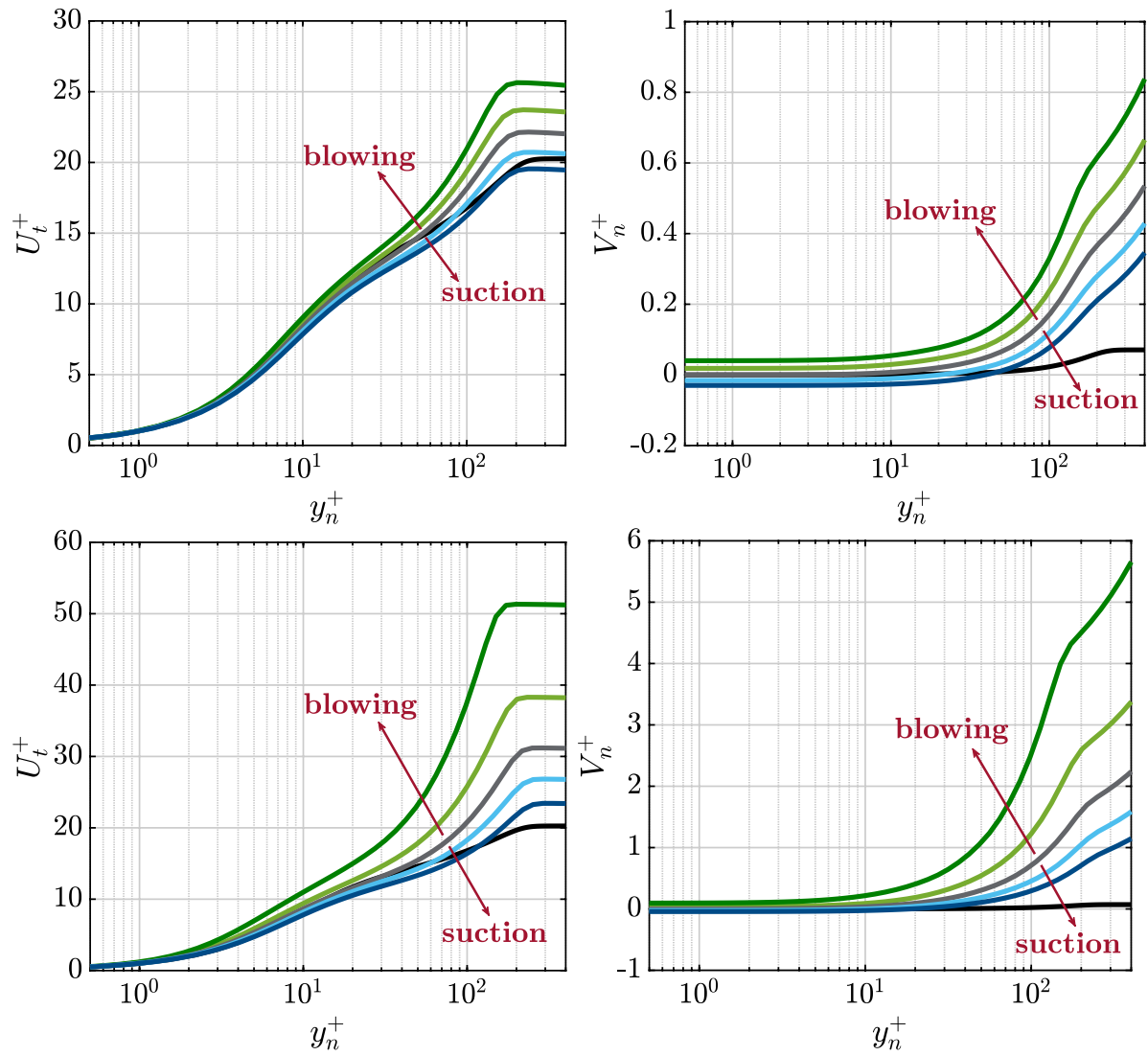

Fig. 5 Inner-scaled profiles of (left) wall-tangential and (right) wall-normal mean velocity components over the suction side for cases A, B, C, D, and E (color code as in Table 1). Top and bottom at $x / c=0.5$ and $x / c=0.8$, respectively. Black lines for reference data of ZPG TBL at similar Reynolds number (Eitel-Amor et al. 2014)

wall-normal velocity component of the mean velocity, $U_{t}^{+}$and $V_{n}^{+}$, as a function of the wall-normal distance, $y_{n}^{+}$, for Cases A, B, C, D, and E. Note that the local wall-shear stress for each case is used to determine the local viscous units. We consider two different locations over the suction side, $x / c=0.5$ and $x / c=0.8$, which differ both in Reynolds number and pressure gradient. The same quantities for a ZPG TBL at similar Reynolds number are also reported as reference (Eitel-Amor et al. 2014).

To characterize the TBL we consider the Reynolds number based on the momentum thickness, $R e_{\theta}=U_{\infty} \theta / \nu$, and the Clauser pressure-gradient parameter $\beta=\delta^{*} / \tau_{w} \mathrm{~d} P_{e} / \mathrm{d} x$. We evaluated $\delta_{99}$ (i.e., the $99 \%$ boundary-layer thickness) using the diagnostic scaling, as in Ref. Vinuesa et al. (2016), which is employed to compute the momentum thickness $\theta$, the displacement thickness $\delta^{*}$, and the pressure gradient at the the boundary-layer edge $\mathrm{d} P_{e} / \mathrm{d} x$. It is important to note that the local values of $\beta$ and $R e_{\theta}$ are not enough to determine the state of the TBL, due to history effects (Bobke et al. 2017). Nevertheless, they give a general indication of the relative strength of the pressure gradient and are useful to perform comparisons. 
In Case A, at $x / c=0.5$ (Fig. 5, top), $\operatorname{Re}_{\theta}=590$ and $\beta=0.9$, and at $x / c=0.8$ (Fig. 5, bottom), $R e_{\theta}=1,150$ and $\beta=6.6$. The ZPG data considered are at $R e_{\theta}=550$. Note that the increase of $R e_{\theta}$ over the suction side is due to both the streamwise development and the APG. For comparison, at $x / c=0.8$ over the pressure side, subjected to a mild FPG, $\operatorname{Re}_{\theta}=465$.

Due to the APG, the inner-scaled velocity in the wake region of the boundary layer is higher than for the reference ZPG and, at the same time, the wall-normal convection is stronger. The effects of uniform blowing and uniform suction on the inner-scaled velocity profiles are similar to those of APG and FPG, respectively, as already pointed out in Ref. Vinuesa and Schlatter (2017). In particular, uniform blowing leads to an even higher velocity in the wake region and stronger wall-normal convection, and uniform suction does the opposite. These effects are more pronounced at $x / c=0.8$ than at $x / c=0.5$, i.e. where the APG is more intense, both for $U_{t}^{+}$and $V_{n}^{+}$. Additionally, compared with pressuregradient effects, blowing and suction have a stronger impact on the buffer-layer region, a region where the profiles of $U_{t}^{+}$and $V_{n}^{+}$from the controlled cases do not agree with those of Case $\mathrm{A}$ and the ZPG reference. This fact is evident for $V_{n}^{+}$, the value of which in the inner layer matches that of the boundary condition at the wall. It is also possible to observe that the changes in the velocity profiles are more pronounced for blowing than for suction for the same control intensity, and that the effects of changing the amplitude are not linear, i.e. twice of the blowing amplitude corresponds to more than twice the difference of $\mathrm{V}^{+}$ between the control and reference cases. Note that this fact is not solely due to the lower friction in the case of stronger blowing, because the skin-friction reduction is proportionally smaller for higher blowing intensity.

In Fig. 6 we show the $U_{t}^{+}$and $V_{n}^{+}$profiles over the pressure side for Cases $\mathrm{F}, \mathrm{G}$ and $\mathrm{H}$ at $x / c=0.8$. The curves for Cases $\mathrm{F}$ and $\mathrm{G}$ are overlapping, despite the slight difference in the control region over the pressure side and uniform suction applied over the suction side in Case F. The TBL over the pressure side is subjected to a moderate FPG (at $x / c=0.8$, $\beta=-0.1$ in Case A) and the impact of uniform blowing is weaker than over the suction side. In particular, the wall-normal convection remains much lower than over the suction
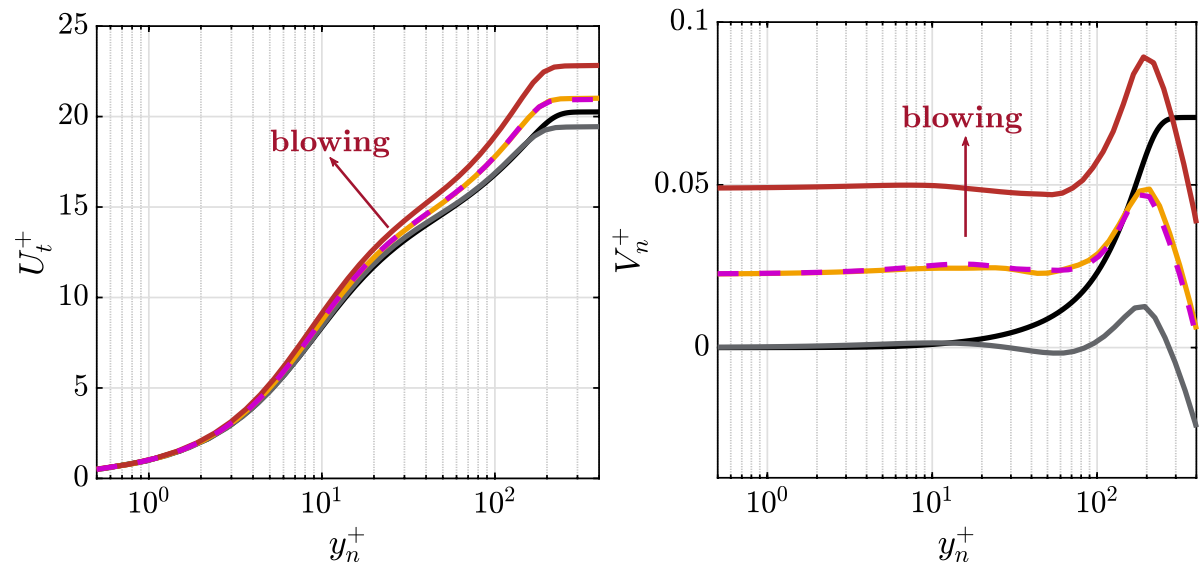

Fig. 6 (Left) Inner-scaled profiles of wall-tangential and (right) wall-normal mean velocity components at $x / c=0.8$ on the pressure side for cases $\mathrm{A}, \mathrm{F}, \mathrm{G}$ and $\mathrm{H}$ (color code as in Table 1). Black lines for reference data of ZPG TBL at similar Reynolds number (Eitel-Amor et al. 2014) 
side in the outer region of the boundary layer, despite the fact that the blowing intensity is the same as in Cases B and C.

In Fig. 7, we show the wall-tangential velocity profiles scaled according to Stevenson's law, originally formulated as a generalization of the logarithmic law for turbulent boundary layer with suction by Stevenson (1963), and recently re-derived by Vigdorovich (2016). According to Stevenson's law, in the logarithmic region, the wall-tangential mean velocity follows the expression:

$$
\frac{2 U_{t}^{+}}{\sqrt{1+V_{w}^{+} U_{t}^{+}}+1}=\frac{1}{K}\left(\ln y^{+}+C_{0}-C_{1} V_{w}^{+}\right)+O\left(y^{+\alpha}\right),
$$

where $V_{w}^{+}$is the value of the inner-scaled wall-normal velocity at the wall, $\alpha<0, K$ and $C_{0}$ are the two constant of the logarithmic law and $C_{1} \simeq 3.5$. Note that, due to the relatively low Reynolds number and intense adverse pressure gradient, the logarithmic region of the profile is virtually absent on the suction side at the considered location. Thus, it is no surprise that the scaled velocity $2 U_{t}^{+} / \sqrt{1+V_{w}^{+} U_{t}^{+}}+1$ does not follow the logarithmic law either. However, it is interesting to observe how the profiles of the control cases collapse quite well on top of the reference, i.e. the scaling (3) appears to capture the effects of blowing and suction. In particular, there is perfect agreement among cases A, B, D and E. Case $\mathrm{C}$ exhibits a slightly different behaviour, which probably relates to the fact that the boundary layer is approaching the condition of mean separation.

We observe similarities between control and pressure-gradient effects on the turbulent fluctuations as well. Figure 8 shows the inner-scaled and outer-scaled profiles of the streamwise velocity fluctuations, $\overline{u u}$, in the streamwise location $x / c=0.8$ on the side of the airfoil where the control is applied.

Note that the outer-scaled profiles are computed using the wall-tangential mean velocity at $\delta_{99}$, denoted $U_{e}$. The comparison between the $\overline{u^{\prime} u^{\prime}}$ and $\overline{u^{\prime} u^{\prime}}$ profiles of Case A and
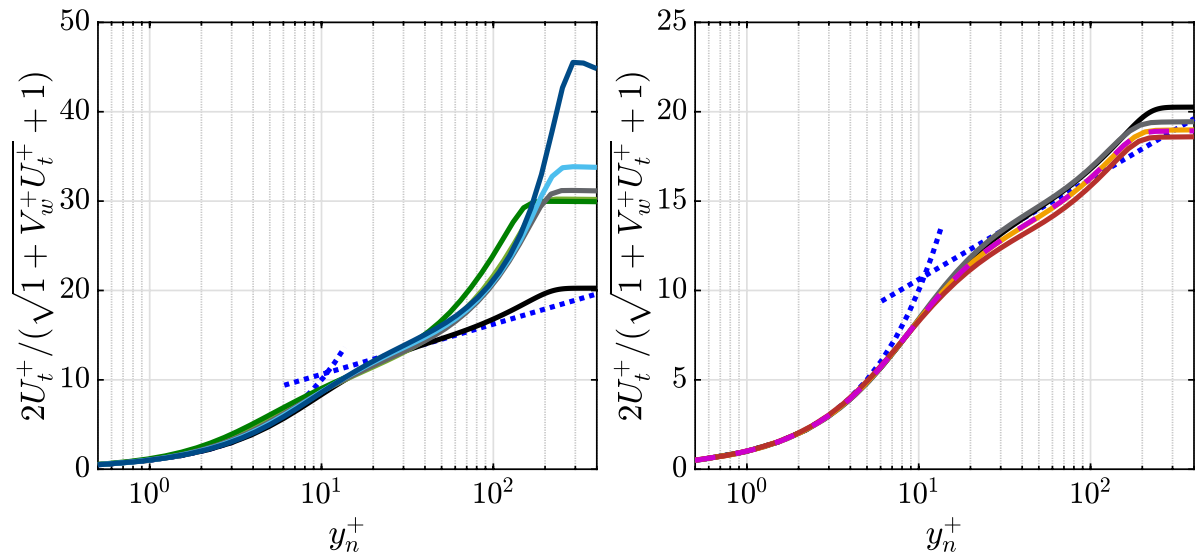

Fig. 7 Profiles of the mean tangential velocity scaled following the Stevenson law (Vigdorovich 2016) at $x / c=0.8$ on (left) the suction side for Cases A, B, C, D and E suction and the (right) pressure side for Case A, F, G and H. Black lines for reference data of ZPG TBL at similar Reynolds number (Eitel-Amor et al. 2014), dotted lines for law of the wall $\left(U_{t}^{+}=y^{+}\right.$and $U_{t}^{+}=1 / K\left(\ln y^{+}+C\right)$, where $C=2.05$ and $\left.K=0.41\right)$ and color code for the cases as in Table 1 

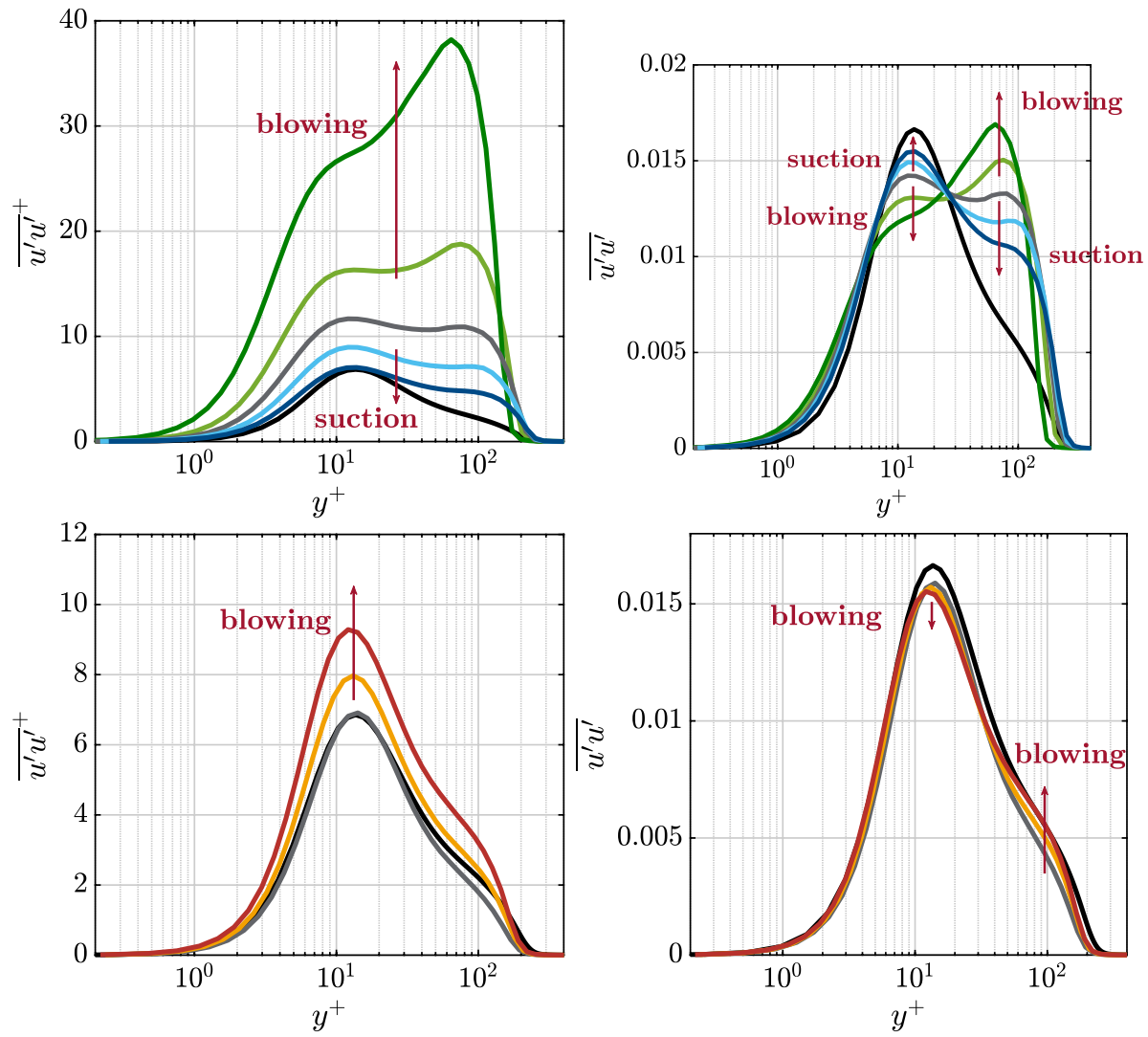

Fig. 8 (Left column) Inner- and (right column) outer-scaled profiles of the streamwise velocity fluctuations at $x / c=0.8$ on (top) the suction side for Cases A, B, C, D, and E and on (bottom) the pressure side for and Cases A, G and H. Black lines for reference data of ZPG TBL at similar Reynolds number (Eitel-Amor et al. 2014) and color code for the cases as in Table 1

the ZPG on the suction and the pressure sides illustrates the well-known impact of a strong adverse pressure gradient and a moderate FPG, respectively. On the suction side, the innerscaled fluctuations are stronger than in the ZPG reference at all wall distances, and a second peak appears farther from the wall. However, inspecting the outer-scaled profiles, which are not affected by the inherently lower friction velocity due to the APG, it is possible to observe that the fluctuations are not always more intense than in the ZPG. Instead, the inner peak is lower in the APG case, and only the second peak is higher than the ZPG value at the corresponding wall-normal location. On the pressure side, since the FPG is quite weak, its effects are almost negligible. Nonetheless, it is possible to appreciate that the fluctuations have a lower intensity than in the ZPG, as expected. On both sides of the airfoil, uniform blowing and suction have effects similar to those of a stronger APG or an FPG, respectively. Uniform blowing leads to higher $\overline{u^{\prime} u^{\prime}}+$ at all wall distances, as a consequence of the lower friction velocity, while the inner peak of the $\overline{u^{\prime} u^{\prime}}$ is lower than in the case without control. On the contrary, uniform suction leads to lower ${\overline{u^{\prime} u^{\prime}}}^{+}$, corresponding to a higher inner peak and a lower outer peak for $\overline{u^{\prime} u^{\prime}}$. On the pressure side, similarly to what we discussed for the mean components of the velocity, the control effects are less 

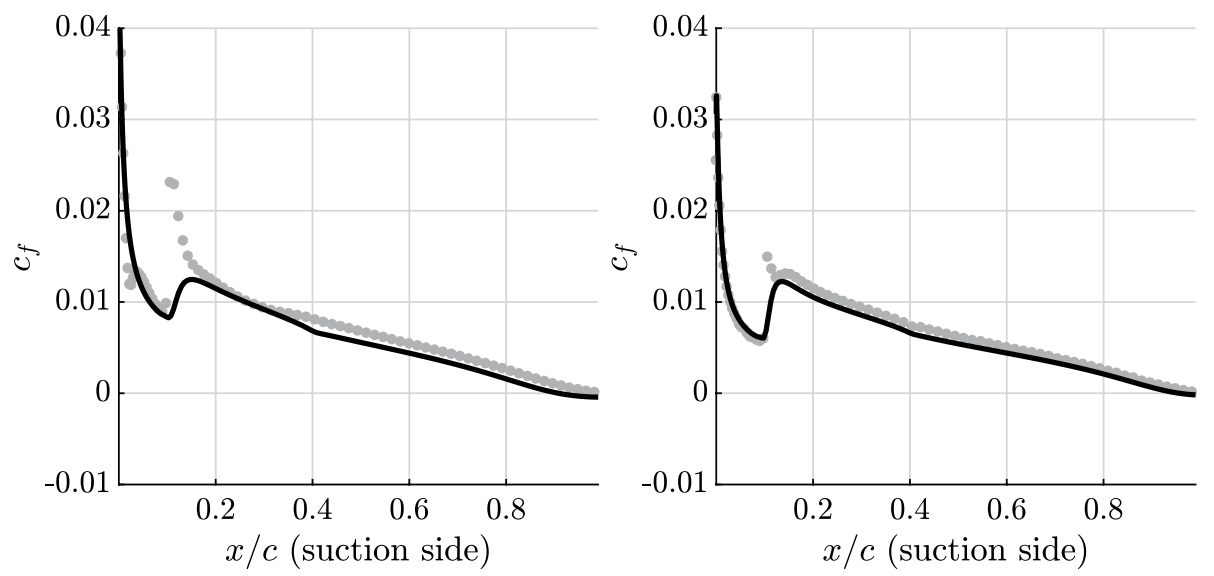

Fig. 9 Skin-friction coefficient over the suction side for (black solid lines) RANS and (grey symbols) LES, at (left) $R e_{c}=200,000$ and (right) $R e_{c}=400,000$

Table 3 Comparison between integrated lift $\left(C_{l}\right)$, skin-friction $\left(C_{d, f}\right)$, pressure $\left(C_{d, p}\right)$ and total drag $\left(C_{d}\right)$, as well as aerodynamic efficiency $(L / D)$, for LES and RANS

\begin{tabular}{llllll}
\hline & $C_{l}$ & $C_{d, f}$ & $C_{d, p}$ & $C_{d}$ & $L / D$ \\
\hline LES $\left(R e_{c}=200,000\right)$ & 0.867 & $0.0128\left(60 \% C_{d}\right)$ & $0.0087\left(40 \% C_{d}\right)$ & 0.0215 & 41 \\
RANS $\left(R e_{c}=200,000\right)$ & 0.851 & $0.0113\left(58 \% C_{d}\right)$ & $0.0083\left(42 \% C_{d}\right)$ & 0.0196 & 43 \\
RANS deviation & $-2 \%$ & $-12 \%$ & $-5 \%$ & $-9 \%$ & $+5 \%$ \\
LES $\left(R e_{c}=400,000\right)$ & 0.902 & $0.0109\left(63 \% C_{d}\right)$ & $0.0063\left(37 \% C_{d}\right)$ & 0.0172 & 52 \\
RANS $\left(R e_{c}=400,000\right)$ & 0.888 & $0.0100\left(60 \% C_{d}\right)$ & $0.0067\left(40 \% C_{d}\right)$ & 0.0167 & 53 \\
RANS deviation & $-2 \%$ & $-8 \%$ & $+6 \%$ & $-3 \%$ & $+2 \%$ \\
\hline
\end{tabular}

significant than over the suction side. In particular, despite the evident modification of the inner-scaled profiles, which is a direct consequence of the lower friction velocity due to blowing, the effects on $\overline{u^{\prime} u^{\prime}}$ are almost negligible. These facts confirm that the TBL subjected to an intense APG is more sensitive to this type of control.

\subsection{Reynolds-Number Dependence of Skin-Friction and Pressure Drag}

Since it is impossible to investigate the control effect at Reynolds numbers typical of applications using LES, we performed RANS simulations for the uncontrolled case in order to assess the evolution of skin-friction and pressure contributions to the total drag with increasing value of Reynolds number. To validate the RANS simulations, and thus assess the fidelity of the predictions, we compare the streamwise development of the skin friction and the integral values of lift, skin-friction and pressure drag with those obtained with LES for two Reynolds numbers, namely $R e_{c}=200,000$ (Case A), and $R e_{c}=400,000$ (Vinuesa et al. 2018) (Fig. 9, Table 3).

The comparison of the $c_{f}$ curves shows a good agreement; the transition location in the RANS is approximately the same as in the LES, although the skin friction is slightly lower in the turbulent region for the RANS data. The deviation in the integrated values 
of the total drag is below $10 \%$ for $R e_{c}=200,000$ and below $5 \%$ for the $R e_{c}=400,000$. Unfortunately, at $R e_{c}=200,000$, such discrepancy is comparable with effects of the various control strategies. This deviation also translates into a difference in the relative proportions of $C_{d, f}$ and $C_{d, p}$ between RANS and LES. At the same time, the agreement is better at higher Reynolds number, and the RANS simulation captures the general trends observed in the LES. In particular, as the Reynolds number increases, $C_{l}$ increases and $C_{d}$ decreases, which results in higher $L / D$, and the skin-friction contributions $C_{d_{f}}$ to the total drag becomes more important, as expected.

Figure 10 illustrates the evolution of $C_{d}, C_{d, f}$, and $C_{d, p}$, as well as the relative proportions of $C_{d, f}$ and $C_{d, p}$ with respect to $C_{d}$ as a function of Reynolds number.

The trends previously mentioned are still observable as the Reynolds number further increases. However, above $R e_{c} \approx 10^{6}$, the RANS results indicate that the relative contributions of friction and pressure drag appear to converge to $\approx 60 \%$ and $\approx 40 \%$ of the total drag, respectively, in agreement with (Schrauf 2005). Based on these results, we can formulate an educated guess of the control effect at higher Reynolds numbers. In particular, if the relative contribution of the pressure to the total drag remains nonnegligible, applying uniform blowing over the suction side will probably result in higher $C_{d}$. This hypothesis is in agreement with the experimental results reported so far in the literature, up to $R e_{c} \approx 1,600,000$ (Eto et al. 2019). Since uniform blowing applied over the suction side also reduces $C_{l}$, this configuration appears to be the least promising. With similar reasoning, we can hypothesize that uniform suction applied over the suction side can remain advantageous at higher Reynolds numbers in terms of $L / D$. In fact, it both improves lift and has positive effects on the pressure drag, which can potentially mitigate the negative impact on the skin friction as long as the two contributions are of similar order of magnitude. The effectiveness of uniform suction, however, will probably decrease as the Reynolds number increase. If these hypotheses are correct, the most promising control configuration for high Reynolds numbers among the ones considered here is the uniform blowing applied over the pressure side. This control configuration reduces the skin friction without having a significant impact on the pressure drag, while it also has a positive effect on the lift.
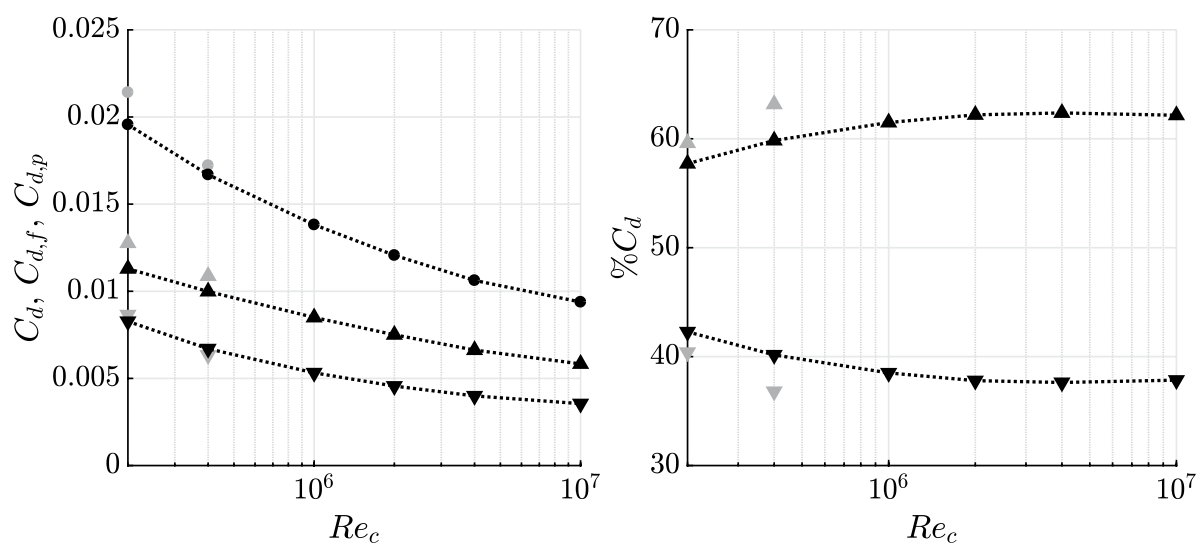

Fig. 10 Reynolds-number dependency of $(\bullet)$ total drag $\left(C_{d}\right),(\boldsymbol{\Delta})$ skin friction $\left(C_{d, f}\right)$, and $(\boldsymbol{\nabla})$ pressure drag $\left(C_{d, p}\right)$. Grey and black colours denote LES and RANS, respectively. Integrated values are shown in the left panel, while the relative contributions to the total drag are shown in the right one 
Note that these deductions rely on the assumption that the control effect will not change dramatically at higher Reynolds numbers. Let us consider, for example, the case of uniform suction. If the skin-friction increase and the pressure-drag reduction both decrease, conclusions drawn at $R e_{c} \approx 200,000$ will be qualitatively valid at higher Reynolds numbers. On the other hand, if, e.g. only the pressure-drag reduction decreases, a different scenario will occur. The most relevant open question for assessing the usefulness of low-Reynolds-number data for high-Reynolds-number prediction is whether the control effects on the different contributions to the total drag will change similarly or not.

\section{Conclusions}

In this study we describe a set of well-resolved LES simulations performed to investigate the effects of uniform blowing and uniform suction on the aerodynamic efficiency $(L / D)$ of a NACA4412 airfoil. We considered four different configurations: (1) uniform blowing applied over the suction side, (2) uniform suction applied over the suction side, (3) uniform blowing applied over the pressure side and (4) uniform suction over the suction side in conjunction with uniform blowing over the pressure side. For configurations (1), (2) and (3) we considered two different blowing/suction intensities, namely $0.1 \%$ and $0.2 \%$ of the incoming free-stream velocity. Including the reference, we conducted a total of 8 wellresolved LES for a Reynolds number based on the chord length of $\operatorname{Re}_{c}=200,000$.

In addition, we carried out RANS simulations up to $R e_{c}=10,000,000$ to describe how the relative skin-friction and pressure contributions to the drag evolve as a function of $R e_{c}$.

Our results regarding the modification of $L / D$ can be summarized as follows: (1) Uniform blowing applied over the suction side is not beneficial in terms of the overall aerodynamic efficiency. In fact, despite the fact that it reduces the local skin friction, it increases the pressure drag and ultimately leads to higher total drag. Furthermore, it decreases lift. (2) Uniform suction applied over the suction side is beneficial for the aerodynamic efficiency, at the present Reynolds number. Although it increases the local skin friction, it also decrease the pressure drag by a sufficient amount to reduce the total drag, and it increases lift. The case with this configuration with a suction intensity of $0.2 \% U_{\infty}$ has the highest aerodynamic efficiency among those considered in the present study, i.e. approximately $11 \%$ higher than that of the reference case. (3) Uniform blowing applied over the pressure side is beneficial for the aerodynamic efficiency since it reduces the skin friction and thereby the total drag, with small modifications of the pressure drag and lift. (4) Uniform suction over the suction side in conjunction with uniform blowing over the pressure side is also beneficial for the aerodynamic efficiency, as expected, since both actuations are beneficial when applied separately. These results are qualitatively in agreement with those of recent experimental studies carried out at higher Reynolds number with similar configurations (Eto et al. 2019; Kornilov et al. 2019).

As already reported in the literature (Kametani and Fukagata 2011; Kametani et al. 2015; Vinuesa and Schlatter 2017), uniform blowing has effects on the turbulent statistics that are similar to those of adverse pressure gradients, and uniform suction has an effect similar to that of favourable pressure gradients. For instance, the former enhances the wallnormal convection, and the latter reduces it. However, we observed that the strong adverse pressure gradient over the suction side amplifies the effects of blowing and suction, while 
the still mild favourable pressure gradient over the pressure side attenuates them. This is the reason why uniform blowing has such a different effect over the suction and pressure sides. Furthermore, we observed that favourable pressure gradients reduce the downstream impact of uniform blowing.

It is critical to understand to what extent the results of studies such as the present one are relevant at higher Reynolds numbers, closer to practical applications. Blowing and suction interact with pressure gradients, and pressure-gradient effects are in general dependent on the Reynolds number and the flow history (Bobke et al. 2017; Sanmiguel Vila et al. 2017; Vinuesa et al. 2018). Due to these facts, it is challenging to assess control performance over a broader range of Reynolds numbers. However, the results of RANS simulations indicate that the relative importance of the pressure contribution to drag will decrease, but remain not negligible up to at least $R e_{c}=10,000,000$. If we assume that the qualitative effects of the control will not change with Reynolds number, e.g. uniform blowing over the suction side will always increase the pressure drag and uniform suction will decreases it, this fact suggests that their effects over the ratio $L / D$ will be similar to those report here. If this hypothesis is correct, the most promising configuration among the ones considered in the present study is uniform blowing applied over the pressure side. On the other hand, if operating at zero net-flow rate is regarded as an advantage (or even necessity), uniform suction applied over the suction side in conjunction with uniform blowing over the pressure side is also recommended.

On a more general perspective, our results underline the need for employing test cases as realistic as possible to assess the effectiveness of the control strategy at hand. Indeed, the overall impact of the control over the total drag, the "side" effects on the lift and the interactions with pressure gradients cannot be easily predicted using results on channel flows or even ZPG TBL.

Finally, we believe that future research should aim at refining RANS predictions, also by taking into account the efficiency losses due to an actual implementation of the actuators, including both the influence of the necessary power for the actuation, and the consideration of the imperfection of the porous plates.

Acknowledgements Open access funding provided by Royal Institute of Technology. This study was funded by the Swedish Foundation for Strategic Research, project "In-Situ Big Data Analysis for Flow and Climate Simulations" (Ref. Number BD15-0082), by the Knut and Alice Wallenberg Foundation and by the Swedish Research Council (VR). The simulations were performed on resources provided by the Swedish National Infrastructure for Computing (SNIC) and within the project CWING on the national supercomputer Cray XC40 Hazel Hen at the High Performance Computing Center Stuttgart (HLRS). We acknowledge that the inclusion of the Stevenson-scaled profiles was suggested by one of the Referees.

\section{Compliance with Ethical Standards}

\section{Conflict of interest The authors declare that they have no conflict of interest.}

Open Access This article is licensed under a Creative Commons Attribution 4.0 International License, which permits use, sharing, adaptation, distribution and reproduction in any medium or format, as long as you give appropriate credit to the original author(s) and the source, provide a link to the Creative Commons licence, and indicate if changes were made. The images or other third party material in this article are included in the article's Creative Commons licence, unless indicated otherwise in a credit line to the material. If material is not included in the article's Creative Commons licence and your intended use is not permitted by statutory regulation or exceeds the permitted use, you will need to obtain permission directly from the copyright holder. To view a copy of this licence, visit http://creativecommons.org/licenses/by/4.0/. 

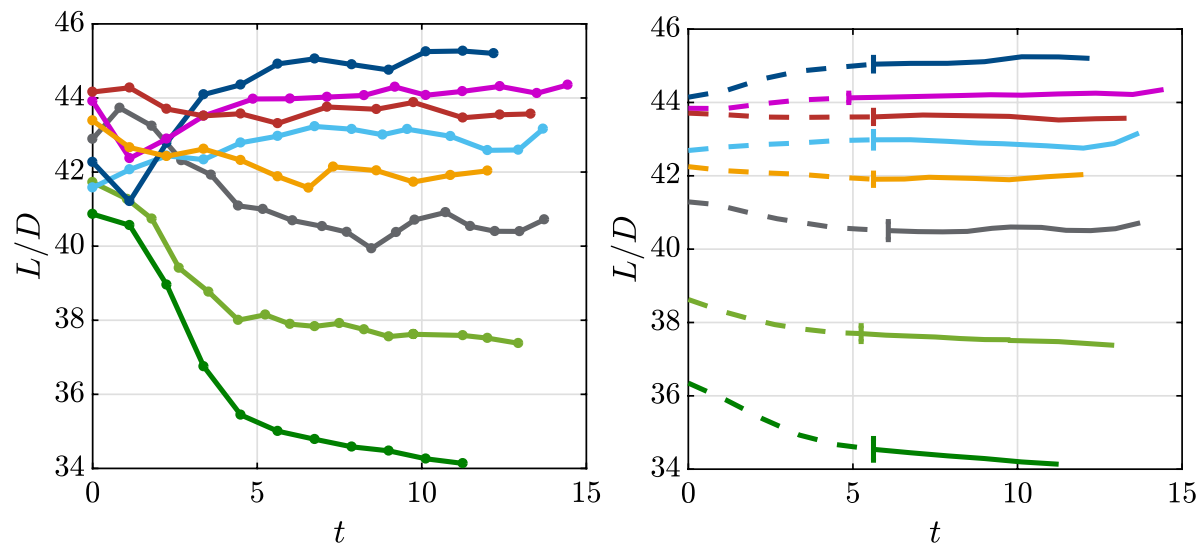

Fig. 11 Aerodynamic efficiency computed based on (left) time average over each single simulation and (right) backward-cumulative averages as defined in Eq. (4). The solid lines in the right panel represent the time interval considered in the global average. Color code as in Table 1

\section{Appendix 1: Statistical Convergence}

The pressure distribution, which contributes significantly to lift and total drag, is sensitive to the development of the wake downstream the airfoil. Subsequently, all integral quantities exhibit a transient behaviour, which can affect the statistics and lead to a much longer time to convergence if included in the time average. It is difficult to predict the length of the transient, and we observed that it varies between the different cases. To illustrate this phenomenon, we show in Fig. 11 (left) the aerodynamic efficiency averaged over approximately a single flow-over time as a function of the simulation time, $t$. Each point in the plot represents a single simulation, and $t$ is the average between its beginning and end times. The transient in Case $\mathrm{A}$ is a consequence of the increasing polynomial order mentioned in Sect. 2, while that in the control cases is due to the activation of the control.

We selected the time interval to perform the global average, with the following procedure. Firstly, we compute a backward-cumulative average, defined as the average between the mean field obtained with each simulation starting from the last one, and weighted with the corresponding averaging time. The backward-cumulative average $\langle X\rangle$ of a certain quantity $X$ is computed as:

$$
\langle X\rangle_{M}=\frac{1}{\Delta t} \sum_{i=M}^{N} \Delta t_{i} X_{i},
$$

where $X_{i}$ is the average value of $X$ computed for $i$ th simulation, $\Delta t_{i}$ is the corresponding average time, $N$ is the total number of simulations carried out for the considered case and $\Delta t=\sum_{i=M}^{N} \Delta t_{i}$. Secondly, we compute the aerodynamic efficiency based on the backwardsaveraged mean fields. The time $t^{*}$ considered for the statistics is that which guarantees the smallest differences between the values of $L / D$ computed with averages between two consecutive times. The aerodynamic efficiency evaluated using the backwards-averaged mean fields is shown in Fig. 11 (right). The time intervals selected to perform the global 
average are indicated with solid lines. The error bars are computed as the standard deviation, defined as:

$$
\sigma_{M^{*}}=\sqrt{\frac{1}{\Delta t} \sum_{i=M^{*}}^{N} \Delta t_{i}\left(X_{i}-\langle X\rangle_{M}\right)^{2}},
$$

where $M^{*}$ is simulation corresponding to $t^{*}$. The trends observed in Fig. 11 suggest that the results for Case $\mathrm{C}$ may still be affected by the initial transient. This simulation was interrupted due to the low $L / D$ and occurrence of mean separation close to the trailing edge.

\section{Appendix 2: Momentum Balance}

We computed the lift and drag coefficients reported in the present paper integrating the pressure and viscous forces over the airfoil surface, as described in Sect. 3.2. An alternative method consists of considering the momentum balance over a control volume surrounding the airfoil. Assuming that conservation of momentum in the steady state holds, the forces applied over the airfoil are equal to the sum of the momentum fluxes across the surface of the volume and the forces applied to it. Discrepancies between the two methods are an indication of the uncertainty of the results, either due to transients, resolution or other effects not captured by the integral equations. In the present study, we consider a control volume extending between $x=-0.25 c$ and $x=1.25 c$, and $y=-0.25 c$ and $y=0.25 c$ in the horizontal and vertical direction, respectively. The vertical and horizontal components of the total force $\boldsymbol{F}=\left(F_{x}, F_{y}\right)$ acting over the control volume are,

$$
\begin{gathered}
F_{x}=\int_{\Lambda} \rho(\boldsymbol{U} \cdot \mathrm{d} \boldsymbol{\lambda}) U-\left(\int_{\Lambda} \rho \overline{u^{\prime} u^{\prime}} \mathrm{d} \boldsymbol{\lambda}\right) \cdot \hat{i}-\left(\int_{\Lambda} \rho \overline{u^{\prime} v^{\prime}} \mathrm{d} \lambda\right) \cdot \hat{i}-\left(\int_{\Lambda} P \mathrm{~d} \boldsymbol{\lambda}\right) \cdot \hat{i} \\
F_{y}=\int_{\Lambda} \rho(\boldsymbol{U} \cdot \mathrm{d} \boldsymbol{\lambda}) V-\left(\int_{\Lambda} \rho \overline{v^{\prime} v^{\prime}} \mathrm{d} \boldsymbol{\lambda}\right) \cdot \hat{j}-\left(\int_{\Lambda} \rho \overline{u^{\prime} v^{\prime}} \mathrm{d} \lambda\right) \cdot \hat{j}-\left(\int_{\Lambda} P \mathrm{~d} \boldsymbol{\lambda}\right) \cdot \hat{j}
\end{gathered}
$$

where $\Gamma$ represents the perimeter of the (bi-dimensional) control volume, $\mathrm{d} \boldsymbol{\lambda}$ is the vector normal to the surface of the volume directed outward, and $\hat{i}$ and $\hat{j}$ are the unitary vectors in the vertical and the horizontal directions. The drag and lift forces are the projections of the total force in the parallel and perpendicular directions to the incoming flow, respectively, and the aerodynamic efficiency is computed subsequently. The comparison of the values of $L / D$ for the two methods is reported in Table 4.

Table 4 Comparison between the aerodynamic efficiency based on drag and lift integrated over the surface of the airfoil and over a control volume

\begin{tabular}{lccc}
\hline Case & $L / D$ (surface int.) & $L / D$ (control vol.) & deviation \\
\hline A & 40.51 & 40.50 & $0.02 \%$ \\
B & 37.70 & 37.77 & $0.19 \%$ \\
C & 34.54 & 34.53 & $0.02 \%$ \\
D & 42.82 & 42.83 & $0.02 \%$ \\
E & 45.04 & 45.03 & $0.03 \%$ \\
F & 44.12 & 44.19 & $0.01 \%$ \\
G & 41.94 & 41.85 & $0.02 \%$ \\
H & 43.64 & 43.56 & $0.02 \%$ \\
\hline
\end{tabular}




\section{References}

Abbas, A., de Vincente, J., Valero, E.: Aerodynamic technologies to improve aircraft performance. Aerosp. Sci. Technol. 28, 100-132 (2013)

Albers, M., Meysonnat, P., Schröder, W.: Actively reduced airfoil drag by transversal surface waves. Flow Turbul. Combust. 102, 865-886 (2019)

Banister, D., Anderton, K., Bonilla, D., Givoni, M., Schwanen, T.: Transportation and the environment. Annu. Rev. Environ. Resour. 36, 247-70 (2011)

Bechert, D.W., Bartenwerfer, M.: The viscous flow on surfaces with longitudinal ribs. J. Fluid Mech. 206, 105-129 (1989)

Bobke, A., Vinuesa, R., Örlü, R., Schlatter, P.: History effects and near equilibrium in adverse-pressuregradient turbulent boundary layers. J. Fluid Mech. 820, 667-692 (2017)

Bottaro, A., Soueid, H., Galletti, B.: Two-equation eddy-viscosity turbulence models for engineering applications. AIAA J. 32, 1598-1605 (1994)

Choi, H., Moin, P., Kim, J.: Direct numerical simulation of turbulent flow over riblets. J. Fluid Mech. 255, 503-539 (1993)

Choi, H., Jeon, W., Kim, J.: Control of flow over a bluff body. Annu. Rev. Fluid Mech. 40, 113-39 (2008)

Dong, S., Karniadakis, G., Chryssostomidis, C.: A robust and accurate outflow boundary condition for incompressible flow simulations on severely-truncated unbounded domains. J. Comput. Phys. 261, 83-105 (2014)

Drela, M.: XFOIL: an analysis and design system for low reynolds number airfoils. In: Brebbia, C.A., et al. (eds.) Low Reynolds Number Aerodynamics, vol. 54, pp. 1-12. Springer, Berlin (1989)

Eitel-Amor, G., Örlü, R., Schlatter, P.: Simulation and validation of a spatially evolving turbulent boundary layer up to Re\$_ltheta $=8300 \$$. Int. J. Heat Fluid Flow 47, 57-69 (2014)

Eto, K., Kondo, Y., Fukagata, K., Tokugawa, N.: Assessment of friction drag reduction on a Clark-Y airfoil by uniform blowing. AIAA J. 57, 2774-2782 (2019)

Fischer, P.F.: An overlapping Schwarz method for spectral element solution of the incompressible Navier-Stokes equations. J. Comput. Phys. 133, 84-101 (1997)

Fischer, P., Lottes, J., Kerkemeier, S.: Nek5000: open source spectral element CFD solver. http://nek50 00.mcs.anl.gov (2008)

Foundation, T.O.: OpenFOAM v7 I Patch releases. https://openfoam.org/news/v7-patch/ (2019)

Fukagata, K., Iwamoto, K., Kasagi, N.: Contribution of Reynolds stress distribution to the skin friction in wall-bounded flows. Phys. Fluids 14, 73-76 (2002)

Gad-el Hak, M.: Flow Control-Passive, Active and Reactive Flow Management. Cambridge University Press, Cambridge (2000)

Hwang, D.: A proof of concept experiment for reducing skin friction by using a micro-blowing technique. NASA pp. TM-107315 (1996)

Hwang, D.: Review of research into the concept of the microblowing technique for turbulent skin friction reduction. Prog. Aerosp. Sci. 40, 559-575 (2004)

Jeong, J., Hussain, F.: On the identification of a vortex. J. Fluid Mech. 285, 69-94 (1995)

Kametani, Y., Fukagata, K.: Direct numerical simulation of spatially developing turbulent boundary layers with uniform blowing or suction. J. Fluid Mech. 681, 154-172 (2011)

Kametani, Y., Fukagata, K., Örlü, R., Schlatter, P.: Effect of uniform blowing/suction in a turbulent boundary layer at moderate Reynolds number. Int. J. Heat Fluid Flow 55, 134-142 (2015)

Kornilov, V.: Current state and prospects of researches on the control of turbulent boundary layer by air blowing. Prog. Aerosp. Sci. 76, 1-23 (2015)

Kornilov, V.I., Boiko, A.V.: Efficiency of air microblowing through microperforated wall for flat plate drag reduction. AIAA J. 50(3), 724-732 (2012)

Kornilov, V., Kavun, I., Popkov, A.: Modification of turbulent airfoil section flow using a combined control action. Thermophys. Aeromech. 26, 165-178 (2019)

Maday, Y., Patera, A.: Spectral element methods for the Navier-Stokes equations. In: Noor, A.K. (ed.) State of the Art Surveys in Computational Mechancis, pp. 71-143. ASME, Paris (1984)

Mahfoze, O.A., Moody, A., Wynn, A., Whalley, R.D., Laizet, S.: Reducing the skin-friction drag of a turbulent boundary-layer flow with low-amplitude wall-normal blowing within a bayesian optimization framework. Phys. Rev. Fluids 4, 094601 (2019)

Menter, F.R.: Two-equation eddy-viscosity turbulence models for engineering applications. AIAA J. 32(8), 1598-1605 (1994)

Menter, F.R., Langtry, R., Völker, S.: Transition modelling for general purpose CFD codes. Flow Turbul. Combust. 77(1-4), 277-303 (2006) 
Menter, F.R., Smirnov, P.E., Liu, T., Avancha, R.: A one-equation local correlation-based transition model. Flow Turbul. Combust. 95(4), 583-619 (2015)

Negi, P., Vinuesa, R., Hanifi, A., Schlatter, P., Henningson, D.: Unsteady aerodynamic effects in smallamplitude pitch oscillations of an airfoil. Int. J. Heat Fluid Flow 72, 378-391 (2018)

Park, J., Choi, H.: Effects of uniform blowing or suction from a spanwise slot on a turbulent boundary layer flow. Phys. Fluids 11(10), 3095 (1999)

Pinkerton, R.: The variation with reynolds number of pressure distribution over an airfoil section. NACA Ann. Rep. 24, 65-84 (1938)

Quadrio, M.: Drag reduction in turbulent boundary layers by in-plane wall motion. Philos. Trans. R. Soc. A 369(1940), 1428-1442 (2011)

Sanmiguel Vila, C., Örlü, R., Vinuesa, R., Schlatter, P., Ianiro, A., Discetti, S.: Adverse-pressure-gradient effects on turbulent boundary layers: statistics and flow-field organisation. Flow Turbul. Combust. 99(3-4), 589-612 (2017)

Savill, A.: A synthesis of T3 test cases predictions. In: Pironneau, O., et al. (eds.) Numerical Simulation of Unsteady Flows and Transition to Turbulence, pp. 404-442. Cambridge University Press, Cambridge (1992)

Schlatter, P., Örlü, R.: Turbulent boundary layers at moderate Reynolds numbers, inflow length and tripping effects. J. Fluid Mech. 710, 5-34 (2012)

Schlatter, P., Stolz, S., Kleiser, L.: LES of transitional flows using the approximate deconvolution model. Int. J. Heat Fluid Flow 25, 549-558 (2004)

Schrauf, G.: Status and perspective of laminar flow. Aeronaut. J. 109, 639-644 (2005)

Stevenson, T.N.: A law of the wall for turbulent boundary layers with suction or injection. CoA Report Aero. No. 166 (1963)

Stroh, A., Hasegawa, Y., Schlatter, P., Frohnapfel, B.: Global effect of local skin friction drag reduction in spatially developing turbulent boundary layer. J. Fluid Mech. 805, 303-321 (2016)

Vigdorovich, I.: A law of the wall for turbulent boundary layers with suction: Stevenson's formula revisited. Phys. Fluids 28, 085102 (2016)

Vinuesa, R., Schlatter, P.: Skin-friction control of the flow around a wing section through uniform blowing. In: Proceedings of European Drag Reduction and Flow Control Meeting (EDRFCM) (2017)

Vinuesa, R., Bobke, A., Örlü, R., Schlatter, P.: On determining characteristic length scales in pressure-gradient turbulent boundary layers. Phys. Fluids 28, 055101 (2016)

Vinuesa, R., Negi, P., Atzori, M., Hanifi, A., Henningson, D., Schlatter, P.: Turbulent boundary layers around wing sections up to Re\$_c $=1\{\} ,000\{\} 000 \$,$$ . Int. J. Heat Fluid Flow 72, 86-99 (2018)$

Viswanath, P.: Aircraft viscous drag reduction using riblets. Prog. Aerospace Sci. 38, 571-600 (2002)

Wood, R.D.: Impact of advanced aerodynamic technology on transportation energy consumpation. SAE Int. 01, 1306 (2004) 